\title{
POLSAR AND POLINSAR MODEL BASED INFORMATION ESTIMATION
}

\author{
Carlos López-Martínez ${ }^{1,2}$, Xavier Fàbregas ${ }^{1,2}$, Luca Pipia $^{1}$ \\ ${ }^{1}$ Universitat Politcnica de Catalunya (UPC), Signal Theory and Telecommunications Dept. (TSC) \\ Remote Sensing Laboratory (RSLab), Barcelona, SPAIN - carlos.lopez@ tsc.upc.edu \\ ${ }^{2}$ Institut d'Estudis Espacials de Catalunya IEEC - CRAE/UPC
}

\begin{abstract}
Speckle for multidimensional SAR data may be modeled as the combination of multiplicative and additive noise sources. As demonstrated, the use of this noise model does not corrupt the estimation of physical information from PolInSAR data. The definition of a model based PolInSAR filter allows also the computation of relative errors for estimated heights of forested areas from PolInSAR data.
\end{abstract}

Index Terms - PolSAR, PolInSAR, Speckle, Filtering

\section{INTRODUCTION}

Radar polarimetry, in the context of Synthetic Aperture Radars (SAR), is a successful technology for the quantitative estimation of biophysical and geophysical parameters of the Earth's surface, especially when combined with coherent scattering modeling. The sensitivity of PolSAR data to the different properties and characteristics of a given surface has made possible the estimation, for instance, of the soil moisture content or the surface roughness. In addition, the penetration capabilities of EM waves, have made possible the study of the internal structure of some targets, for instance, snow or vegetation. The introduction of interferometry, leading to polarimetric SAR interferometry (PolInSAR) has permitted also to include sensitivity to the vertical structure of the target.

When confronted to the study of natural targets, the main observables in PolSAR or PolInSAR are the covariance or the coherency matrices. The aforementioned coherent scattering modeling is applied to these observables, from where the physical parameters of interest are retrieved. Nevertheless, a PolSAR or a PolInSAR system does not directly measure these matrices, but the scattering matrix from where the covariance or the coherency matrices must be estimated. The necessity of this estimation process is due to the presence of the speckle noise component. Consequently, this estimation could be also considered as a speckle filtering process.

Despite there exist satisfactory techniques to cope with speckle noise in PolSAR or PolInSAR data, the problem can

This work has been funded by the Ramon-y-Cajal program and the I+D projects TEC2007-65690/TCM and TEC2008-06764-C02-01. not be considered satisfactorily solved. Among the different alternatives, special attention must be paid to the important contributions of J.S. Lee, especially [1], and also the work of $R$. Touzi [2]. In [1], on the basis of a multiplicative noise model for the speckle noise component, the authors established a first set of principles a PolSAR filter should follow to avoid an alteration of polarimetric information. The understanding of the speckle noise component for multidimensional SAR data increased with the work reflected in [3], where the authors demonstrated that the previous noise model presents clear limitations to completely describe speckle. These can be overcome considering a multiplicativeadditive speckle noise model for multidimensional SAR data. Based on this assumption, a new PolSAR speckle filtering technique was introduced in [4], where it was shown that the filtering principles introduced in [1] could be relaxed leading to a better estimation of the information of interest.

The advantages of the filtering technique introduced in [4] can be exported to PolInSAR. As it will be shown, to consider the multiplicative-additive speckle noise model leads to a better estimation of the covariance and the coherency matrices. This argument will be extended to the estimation of physical parameters, where it shall be demonstrated that to consider this improved speckle noise model in PolInSAR may contribute to a better estimation of forest parameters. In addition to that, this work will also analyze the statistical properties of the estimators of these physical parameters.

\section{MULTIPLICATIVE-ADDITIVE SPECKLE NOISE MODEL}

In case of homogeneous areas, the measured scattering matrix can be described by the complex, zero-mean Gaussian distribution. Under this set of assumptions, the estimated covariance and coherency matrices can be described according to the complex Wishart distribution. Both statistical models have been widely evaluated and accepted by the research community.

The covariance and coherency matrices are constructed considering the Hermitian product of the different constituting elements of the scattering matrix. Based on this observation, an extended speckle noise model was introduced in 


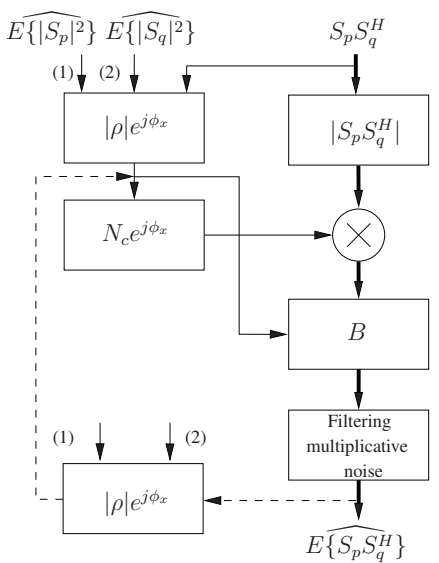

Fig. 1. Processing chain of the MBPolInaSAR filter for the offdiagonal elements of the coherency or coherency matrices.

[3], where it is shown that the Hermitian product of two SAR images may be modeled as

$$
\begin{aligned}
S_{p} S_{q}^{H} & =\psi \bar{z}_{n} N_{c} n_{m} \exp \left(j \phi_{x}\right) \\
& +\psi\left(|\rho|-N_{c} \bar{z}_{n}\right) \exp \left(j \phi_{x}\right) \\
& +\psi\left(n_{a r}+j n_{a i}\right) .
\end{aligned}
$$

In the previous model

$$
N_{c}=\frac{\pi}{4}|\rho|_{2} F_{1}\left(\frac{1}{2}, \frac{1}{2} ; 2 ;|\rho|^{2}\right)
$$

where ${ }_{2} F_{1}(a, b ; c ; z)$ is the Gauss hypergeometric function. We redirect to [3] for a detailed description concerning the parameters of the previous model. The term $n_{m}$ refers to the speckle noise component characterized for presenting a multiplicative noise behavior respect to the information of interest. The term $n_{a r}+j n_{a i}$ is the complex speckle noise component presenting an additive nature respect to the information of interest. Eq. (1) establishes that speckle noise for the complex Hermitian product of SAR images results from the combination of multiplicative and additive noise components. The combination of these components is determined by the complex correlation coefficient $\rho$ that characterizes the Hermitian product $S_{p} S_{q}^{H}$. As observed in the next equation, the multiplicative-additive speckle noise model (1) does not introduce any information loss as

$$
\begin{aligned}
E\left\{S_{p} S_{q}^{H}\right\} & =\psi \bar{z}_{n} N_{c} E\left\{n_{m}\right\} \exp \left(j \phi_{x}\right) \\
& +\psi\left(|\rho|-N_{c} \bar{z}_{n}\right) \exp \left(j \phi_{x}\right) \\
& +\psi E\left\{n_{a r}+j n_{a i}\right\} \\
& =\psi|\rho| \exp \left(j \phi_{x}\right) .
\end{aligned}
$$

The model (1) may be extended to all the elements of the covariance or coherency matrices in case of PolSAR or PolInSAR data. For the diagonal elements it has been demonstrated that the multiplicative-additive speckle noise model reduces to the classical multiplicative model of the intensity. For the off-diagonal elements, speckle results from the combination of multiplicative and additive noise components.

\subsection{Model Based PolInSAR Speckle Noise Filter}

Based on the multiplicative-additive speckle noise model, a new model based technique for speckle noise reduction in PolInSAR data may be introduced. This filtering approach has been already assessed and validated in case of PolSAR data [4]. Based on the reported results, it has been demonstrated that the filtering principles introduced in [1] could be relaxed, leading to a better estimation of the information of interest, without corrupting the polarimetric information.

The following list details the main steps of the model based PolInSAR (MBPolINSAR) filtering approach considered in this work:

1. Separation of elements: The elements of the covariance or coherency matrices are separated into diagonal and off-diagonal elements. Diagonal elements are only affected by the multiplicative speckle noise component, whereas off-diagonal ones are affected by both, the multiplicative and the additive speckle noise components.

2. Diagonal elements processing: Diagonal elements of the covariance or coherency matrices can be processed by any filtering process optimizing multiplicative speckle noise reduction. In the implementation employed in the following, the multilook filtering approach is considered.

3. Off-diagonal elements processing: The speckle noise reduction process of these elements must be done according to (1). The optimum reduction of the multiplicative and the additive speckle components is performed according to the complex correlation coefficient, which is estimated through a multilook process. The availability of the complex correlation coefficient permits to eliminate the additive speckle components, whereas the multiplicative one is filtered considering the same multillok approach to filted the diagonal elements. This process can be iterated as the estimation of the complex correlation coefficient is improved. Details of this filtering process are given in Fig. 1.

4. Reconstruction: The estimated covariance matrix is reconstructed by arranging the processed diagonal and off-diagonal elements in its original form.

As it may be deduced, the implementation of the MBPolInSAR filtering approach considered in this paper focus on the maintenance of the polarimetric information and it does not optimize the maintenance of spatial resolution or details. In this case, it will retain the morphological properties of the native multilook.

\section{FOREST INFORMATION EXTRACTION FROM POLINSAR DATA}

The combination of polarimetric and interferometric sensitivities has been demonstrated successful for the quantitative 


\begin{tabular}{lc}
\hline Range spatial resolution & $1.5 \mathrm{~m}$ \\
Azimuth spatial resolution & $1.5 \mathrm{~m}$ \\
Wavelength $\lambda$ & $0.23 \mathrm{~m}$ \\
Flight height $H$ & $3000 \mathrm{~m}$ \\
Mean incidence angle $\theta_{0}$ & $45 \mathrm{deg}$ \\
\hline
\end{tabular}

Table 1. Flight geometry for simulated PolInSAR data.

analysis of forested regions [5], thanks to introduction a coherent modeling of the coherency matrix [6], referred to as Random Volume over Ground (RVoG) model, able to estimate the following forest parameters: forest height $h_{v}$, underneath topography $\phi_{0}$ and extinction coefficient $\sigma$.

The coherency matrix needs to be estimated for the original data recorded by the SAR system. Some authors have already analyzed the effect of this estimation process on the retried information [7], but mainly focusing on the morphological properties of the filtered data. The aim of this paper is to complement the previous evaluation works. On the one hand, through the quantitative evaluation of the filtering technique proposed in Section 2.1, and on the other hand by assessing the statistical properties of the physical quantities that can be extracted in case of forested areas by means of PolInSAR data.

\section{EVALUATION AND RESULTS}

The most important limitation when facing quantitative evaluations of filtering techniques in the case of multidimensional SAR data, or in the particular case of an exhaustive statistical assessment of the forest physical parameters that may be estimated via [6], is the complete lack of ground truth. This problem can be only circumvented considering simulated SAR data. The simulation of PolInSAR data for forested areas may be performed using different approaches, under the limitation that this simulation must be coherent. The stochastic simulation considered in this work is based on generating PolInSAR data correlated according to the RVoG coherent scattering model specified in [6]. Since data are simulated according to the scattering model in which the inversion procedure for $h_{v}, \phi_{0}$ and $\sigma$ is based on, any deviation of the retrieved information from the nominal or true values may be basically attributed to the speckle noise filtering process, and in a lower degree to the inversion procedure in [6]. The simulation shall be done according to the L-band DLR E-SAR system considering two parallel horizontal tracks, as specified in Table 1. Under the previous geometry, a particular scenario of a vegetated terrain is considered, according to the RVoG model. The volume contribution of the model is simulated according to the following coherency matrix

$$
T_{v}=0.125\left[\begin{array}{ccc}
1 & 0 & 0 \\
0 & 0.25 & 0 \\
0 & 0 & 0.25
\end{array}\right]
$$
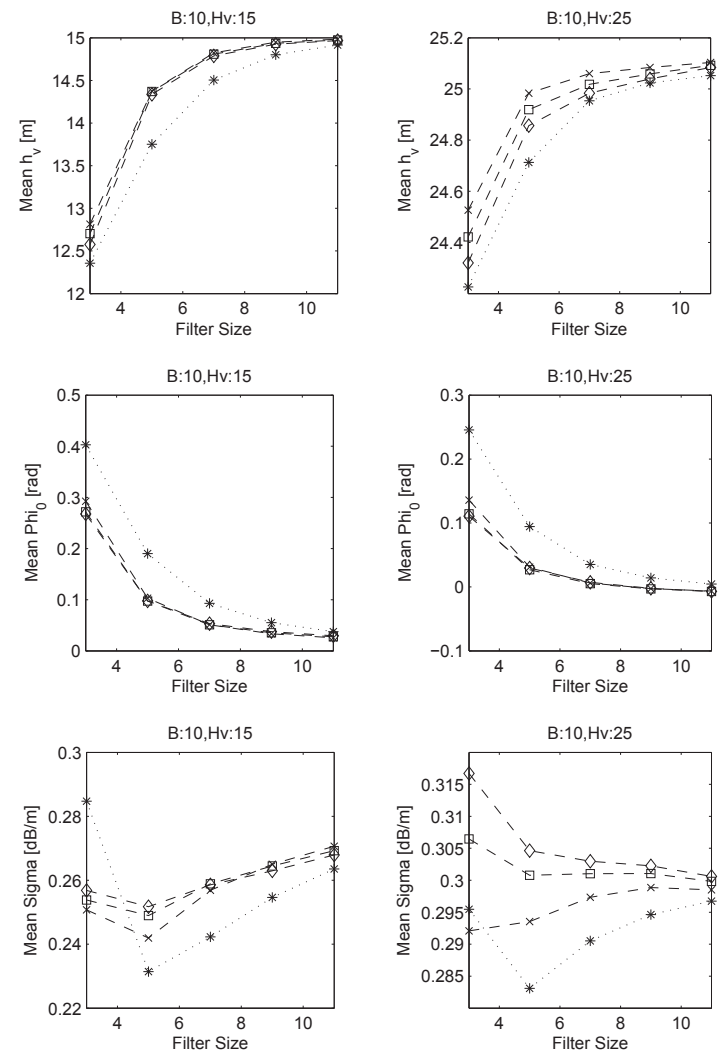

Fig. 2. Estimated mean values of $h_{v}, \phi_{0}$ ( $\left.\mathrm{Phi}_{0}\right)$ and $\sigma$ (sigma). B indicates baseline in $\mathrm{m}$. Hv indicates simulated height in $\mathrm{m}$. Filter Size indicates the Boxcar side dimension. Legend: $(*)$ Multilook, $(\times) 1$ it. MBPolInSAR, $(\square) 2$ it. MBPolInSAR, $(\diamond) 3$ it. MBPolInSAR.

The ground contribution considers a flat, rough, loamy terrain with $2.2 \%$ water content simulated according to the X-Bragg rough surfaces scattering model, as specified in Table 2. Both scattering contributions are related with a nominal groundto-volume ratio of $-5 \mathrm{~dB}$. The different PolInSAR simulated

\begin{tabular}{lc}
\hline Roughness $\beta_{1}$ & $5 \mathrm{deg}$ \\
Ground-to-volume ratio $\mu$ & $-5 \mathrm{~dB}$ \\
Relative permittivity $\varepsilon_{r}$ & 3.5 \\
Ground phase $\phi_{0}$ & $0 \mathrm{deg}$ \\
\hline
\end{tabular}

Table 2. Parameters of the surface scattering contribution of the RVoG coherent scattering model for simulated PolInSAR data according to the X-Bragg model.

datasets employed in the following will follow the previous scenario, considering four different horizontal baseline values of $5,10,15$ and $20 \mathrm{~m}$, and forest heights between 5 and 35 $\mathrm{m}$. Finally, $\phi_{0}$ shall be considered equal to zero in all the simulations and $\sigma=0.3 \mathrm{~dB} / \mathrm{m}$.

All the previous datasets have been generated and filtered with the Multilook and the MBPolInSAR approach detailed in 

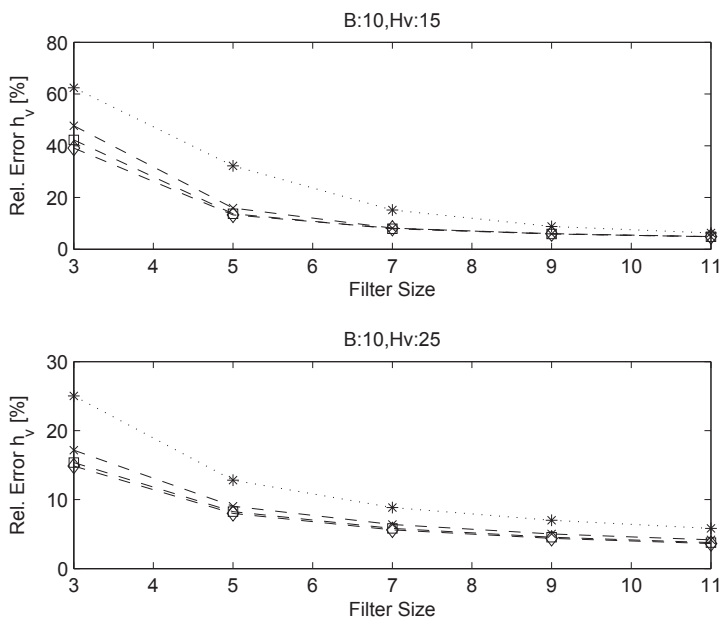

Fig. 3. Relative error of estimated height as a function of the multillok dimensions for a baseline of $10 \mathrm{~m}$ and simulated heights of 15 and $25 \mathrm{~m}$. Same legend as Fig. 2.

Section 2.1. Multilook has been considered with dimensions of $3 \times 3,5 \times 5,7 \times 7,9 \times 9$ and $11 \times 11$ pixels. MBPolInSAR has been considered with the same Multilook dimensions and from 1 to 3 iterations. Fig. 2 shows the plots of the retrieved physical parameters considering [6] for a baseline of $10 \mathrm{~m}$ and a simulated height of 15 and $25 \mathrm{~m}$. In all the cases, one may clearly observe that the MBPolInSAR approach outperforms the retrieval of physical parameters, compared with the multilook, despite to process the elements of the coherent matrix differently, thanks to considering the multiplicative-additive speckle noise model. Forest height and topographic phase are correctly estimated with relatively large averaging windows. It is important to observe that all the physical parameters are asymptotically non-biased with respect to the number of looks. This behavior appears in all the simulations conducted in this study.

Figs. 3 and 4 plot the relative error of the estimated height as a function of the averaging strength and as a function of the simulated height. In all the cases, on may observe that MBPolInSAR outperforms multilook as the former estimates height with lower variances. Height errors below $5 \%$ are only possible for heights above $15 \mathrm{~m}$ and considering large averaging windows $(11 \times 11)$. The error can be decreased if the multiplicative-additive speckle noise model is considered.

\section{CONCLUSIONS}

As demonstrated, the use of the multiplicative-additive speckle noise model to filter PolInSAR does not damage useful information but helps to improve estimation of physical parameters. As demonstrated with the Multilook and the MBPolInSAR filtering approaches, it could be concluded that forest parameters can be considered asymptotically non bi-

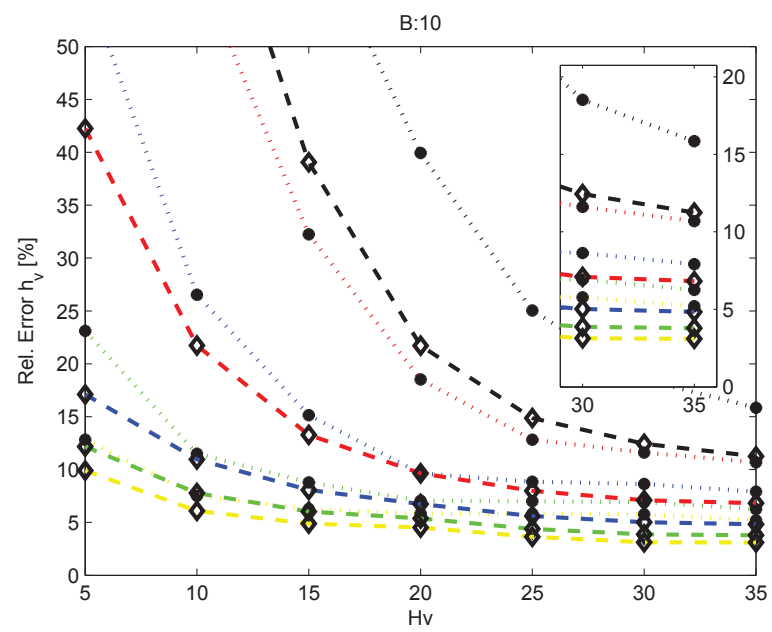

Fig. 4. Relative error of estimated height as a function of the simulated height for a baseline of $10 \mathrm{~m}$. Same legend as Fig. 2, black for $3 \times 3$, red for $5 \times 5$, blue for $7 \times 7$, green for $9 \times 9$ and yellow $11 \times 11$.

ased. Considering simulated PolInSAR data according to the RVoG model, height error below $5 \%$ can only be achieved for heights above $15 \mathrm{~m}$ and a relative hight number of samples, or considering the multiplicative-additive speckle noise model.

\section{References}

[1] Jong-Sen Lee, M.R. Grunes, and G. de Grandi, "Polarimetric SAR speckle filtering and its implication for classification," Geoscience and Remote Sensing, IEEE Transactions on, vol. 37, no. 5, pp. 2363-2373, Sept. 1999.

[2] R. Touzi and A. Lopes, "The principle of speckle filtering in polarimetric SAR imagery," Geoscience and Remote Sensing, IEEE Transactions on, vol. 32, no. 5, pp. 1110 - 1114, Sept. 1994.

[3] C. López-Martínez and X. Fàbregas, "Polarimetric SAR speckle noise model," IEEE Trans. Geosci. Remote Sensing, vol. 41, no. 10, pp. 2232-2242, Oct. 2003.

[4] C. López-Martínez and X. Fàbregas, "Model-based polarimetric sar speckle filter," Geoscience and Remote Sensing, IEEE Transactions on, vol. 46, no. 11, pp. 3894-3907, Nov. 2008.

[5] S. R. Cloude and K. P. Papathanassiou, "Polarimetric SAR interferometry," Geoscience and Remote Sensing, IEEE Transactions on, vol. 36, no. 5, pp. 1551-1565, Sept. 1998.

[6] S.R. Cloude and K.P. Papathanassiou, "Three-stage inversion process for polarimetric SAR interferometry," Radar, Sonar and Navigation, IEE Proceedings -, vol. 150, no. 3, pp. 125-134, 2003.

[7] K.P.; Grunes M.R.; Woodhouse I.H. Jong-Sen Lee; Cloude, S.R.; Papathanassiou, "Speckle filtering and coherence estimation of polarimetric sar interferometry data for forest applications," Geoscience and Remote Sensing, IEEE Transactions on, vol. 41, no. 10, pp. 2254-2263, 2003. 